\title{
Slipped Capital Femoral Epiphysis - A 10-year Review of Cases Treated by Open Reduction and Realignment Osteotomy
}

\author{
Onuoha KM*, Bassey AE, Akinola B, Ekwe KK, Ogedegbe FE, Adedapo A and Onuoha CEO \\ Department of Orthopaedics, Cedarcrest Hospitals, Abuja, Nigeria
}

*Corresponding author: Onuoha KM, Department of Orthopaedics, Cedarcrest Hospitals, Abuja, Nigeria

To Cite This Article: Onuoha KM, Slipped Capital Femoral Epiphysis - A 10-year Review of Cases Treated by Open Reduction and Realignment Osteotomy. 2020 - 7(4). AJBSR.MS.ID.001165. DOI: 10.34297/AJBSR.2020.07.001165.

Received: 紫 February 06, 2020; Published: 眥 February 19, 2020

\begin{abstract}
Background: Slipped capital femoral epiphysis (SCFE) is a common hip disorder affecting adolescents during the period of growth spurt. They typically present with hip pain with or without difficulty in bearing weight and severity is based on the degree of slip radiologically. Moderate to severe slips can be treated with open reduction and realignment osteotomies.
\end{abstract}

Objective: To determine the pattern of presentation of patients with moderate to severe SCFE, treatment offered and outcome.

Methodology: Retrospective Case series of patients presenting over a 10-year period and severity determined radiologically. All patients were treated with open reduction and realignment osteotomies (Fish, Subtrochanteric). Patients were followed up and monitored for complications.

Results: Six patients had unilateral SCFE while one was bilateral making a total of 7 patients (8 hips). One had a Southwick angle of 47 degrees while others had angles over 50 degrees. All were females with a mean age of 12 years, majority (67.5\%) on the left side. The Commonest symptom was hip pain (85.6\%) lasting 3-18 months prior to presentation. All were on crutches and could not weight bear without them. Six were treated with open reduction and subcapital osteotomy while two hips had open reduction with a subtrochanteric osteotomy. All patients were followed up for an average of 22 months and none had avascular necrosis or chondrolysis.

Conclusion: Adolescent Females in our environment present more commonly with severe, unstable, SCFE which can be diagnosed clinically and confirmed radiologically. Prompt Treatment with open reduction and realignment osteotomies via the subcapital or subtrochanteric types affords the best outcomes with minimal risk of feared complications.

Keywords: Slipped Capital Femoral Epiphysis, Open Reduction, Realignment Osteotomy

\section{Introduction}

Slipped Capital Femoral Epiphysis (SCFE) is the commonest hip disorder affecting adolescents [1]. It is characterised by a slippage of the metaphysis superiorly and anteriorly in relation to the epiphysis. This condition is not life-threatening but can result in marked deformity and morbidity if diagnosis is missed or appropriate treatment is not offered.

Its incidence rate is $0.71-10.8 / 100,000$ [1] with the highest rates seen in Blacks, Hispanics and Pacific islanders [2,3]. Majority of slips occur in people in the age group 10-16 years. Rarely, does it occur outside this range, and when it does there is often an underlying predisposing factor such as an endocrinopathy, renal osteodystrophy or radiation exposure. SCFE is commoner in males than in females, and the left hip is more affected than the right [2].

The slip occurs as a result of mechanical overload (obesity) on a vulnerable physis. Weakening of the perichondral ring of LaCroix and unlocking of the physeal mamillary processes are changes that occur during the growth spurt of adolescence. These changes make the physis vulnerable to slip.

Various classification and grading systems exist to help diagnose and guide treatment. Loder et al [4] classified SCFE based on the 
patient's ability to bear weight, on the affected limb. Stable SCFE is one that can bear weight on the limb, while the one who cannot bear weight is said to be unstable. This classification correlates with risk of avascular necrosis. Southwick [5] grades SCFE into mild, moderate and severe based on the angle of slip, with worse slips having larger angles.

Clinical features include hip, thigh or knee pain, limping or inability to walk. Limb shortening, externally rotated attitude and limitation in flexion, adduction and internal rotation are some findings that are usually present.

Diagnosis is usually confirmed on x-rays, which reveals the slip, Southwick angle, radiological indices such as Trethowan's, Steel's and Capener's signs. In chronic cases, there may be avascular necrosis (AVN) of femoral head, secondary osteoarthritis and deformities such as coxa varus. Open reduction though associated with increased risk of AVN has been found to be useful in treating severe cases. This is often combined with a realignment osteotomy to correct residual deformity. Different types of osteotomies have been described, these are: subcapital (Dunn, Fish), basicervical, intertrochanteric and subtrochanteric. The subcapital osteotomies have the greatest capacity to correct SCFE deformities but also carry the highest risk of AVN.
We present a case series of patients who had open reduction and osteotomies done for moderate to severe SCFE at the Cedarcrest Hospitals Abuja, Nigeria over a 10-year period.

\section{Patients and Methods}

The case folders/electronic records of all patients who received treatment at Cedarcrest Hospitals for SCFE from July 1, 2008 to June 30, 2018 were retrieved, and those who had open reduction and realignment osteotomy for severe SCFE were collated and analyzed. Data was collected on the patients demographic details, side involved, laterality, Loder classification and Southwick grade, treatment, implants used, complications and follow-up duration. These were entered into a structured proforma. Clinical outcomes were assessed using the hip range of motion at the follow-up visit, as well as time to full weight bearing. Inclusion criteria were patients who had severe SCFE and were treated by open reduction and realignment osteotomy. Those excluded were patients who were lost to follow up, those who received treatment at other hospitals before arrival, patients with complications before presentation.

\section{Results}

Table 1 below is a summary of the findings during the study period, reflecting the patients' demographic data, clinical presentation, classification, treatment offered and follow up.

Table 1: Summary of results ( $n=8$ hips in 7 patients).

\begin{tabular}{|c|c|c|}
\hline & Finding & Percentage (\%)/Range \\
\hline Mean Age (years) & 12 & $0 / 100$ \\
\hline Sex (M/F) & $0 / 7$ & $37.5 / 67.5$ \\
\hline Side affected (R/L) & $5-$ Mar & 87.5 \\
\hline Presenting complaint & 7 & 12.5 \\
\hline Hip pain & 1 & 100 \\
\hline Knee pain & & 0 \\
\hline Loder Classification & 7 & 100 \\
\hline Unstable & 0 & 0 \\
\hline stable & & \\
\hline Morphological type & 8 & 75 \\
\hline Varus & 0 & 25 \\
\hline Valgus & & Aug-48 \\
\hline Type of osteotomy & 6 & \\
\hline Subcapital & 2 & \\
\hline Subtrochanteric & 22 & \\
\hline Mean follow-up (months) & & \\
\hline
\end{tabular}

\section{Overview}

In the period under review 52 patients were treated for SCFE, of which 10 patients $(19.2 \%)$ had open reduction and realignment osteotomy. One patient had bilateral SUFE making a total of 11 hips. Three patients (3 hips) were excluded as they were lost to follow up. Thus, 7 patients (8 hips) were studied. They were all female, age range 10 and 13 years. Mean age was 12 years. The commonest presenting complaint was hip pain affecting 7 hips (87.5\%) and knee (referred) pain in 1 patient (12.5\%). The duration of symptoms prior to presentation ranged between 3 - 18 months. There were 5 left hips (67.5\%), 2 right (25\%) and 1 was bilateral. There was a history of premorbid trauma in one patient. 


\section{Classification and grade}

All had unstable stable slips as they had to limp or use crutches, also one of which had an unstable slip and presented with knee pain. On Southwick grading, there was one hip with moderate grade while the remaining 7 had severe grades. Southwick angle ranged from $470-700$. The slips were all of varus type.

\section{Treatment/rehabilitation/complications}

6 hips of $8(75 \%)$ had open reduction and subcapital osteotomies while 2 had open reduction and subtrochanteric osteotomies. All subcapital osteotomies were fixed with $6.5 \mathrm{~mm}$ cannulated cancellous screws while the subtrochanteric were fixed with dynamic hip screws. All patients with unilateral SCFE had bilaterally similar hip ROM at the time of last review. One patient had prophylactic contralateral pinning.

All patients achieved full weightbearing within 6 months of surgery, except for one patient who had delayed union and did not achieve same until 11 months post-surgery.

4 hips (50\%) had complications; these included secondary osteoarthritis, coxa varus with LLD, delayed union and screw cutout requiring removal and re-fixation, affecting 1 hip each. There were no cases of AVN or chondrolysis complicating surgery. Mean follow-up duration for all patients was 22 months (range of 8-48 months) (Figure 1).

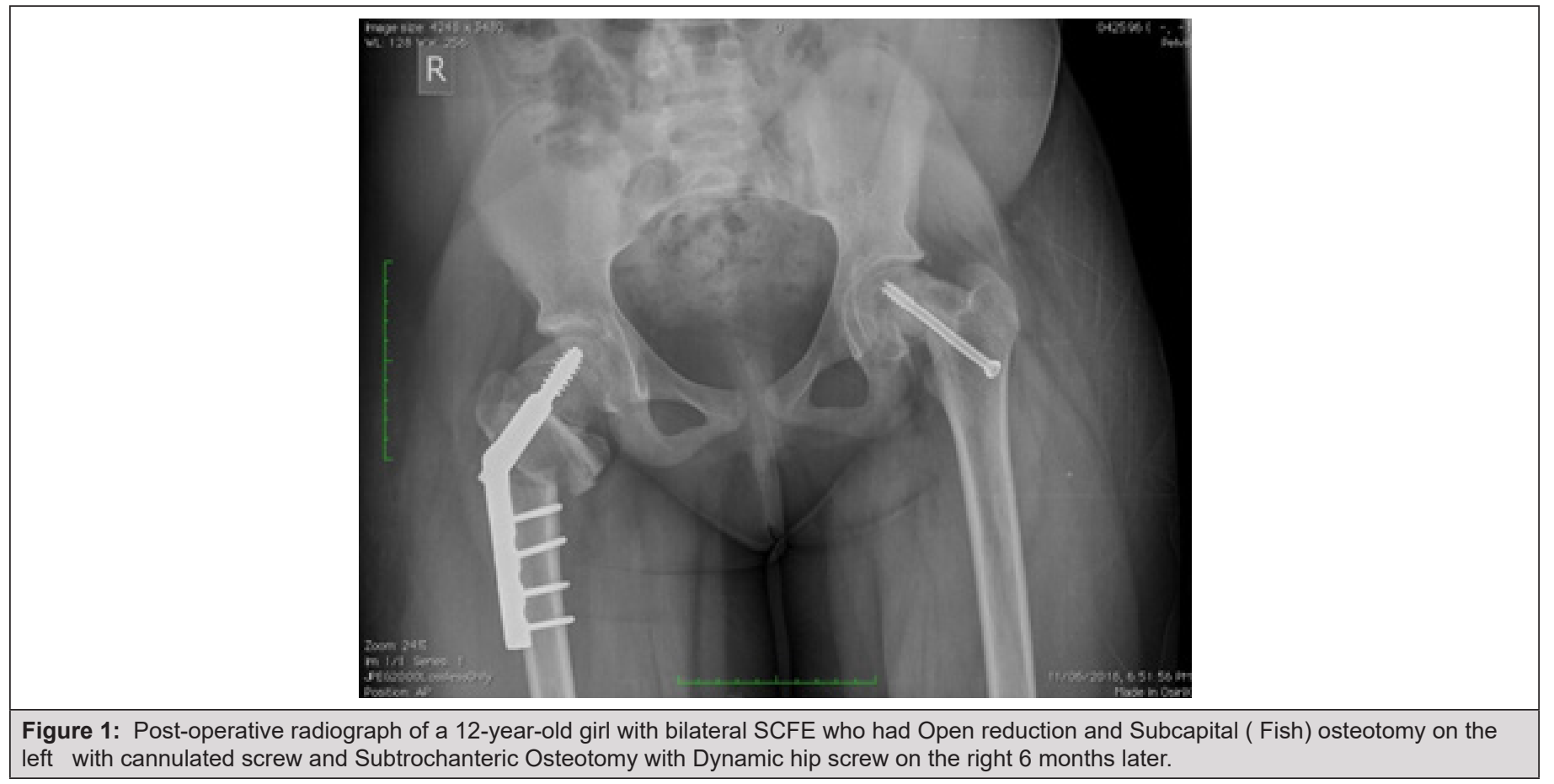

\section{Discussion}

All the patients that had realignment osteotomy in the period under review were female. This is quite different from the general sex distribution of patients with SCFE which reveals a higher incidence in males [1,2]. As well as from a retrospective study on subcapital osteotomies that also reported male preponderance [6]. Yinusa et al in his study in Lagos Nigeria, however, reported a female population that was more than twice the male [7] and similar finding was observed in Katchy's study, though the difference was less [8]. Thus far, the Nigerian studies have shown a female predilection. This could be attributed to females generally having a higher BMI than males in that age group thus predisposing them to more severe slips. Some studies have shown the reversal in sex distribution in special situations, for example, valgus slips were 2 times more likely in females according to Shank's study [9]. Palocaren et al also showed that in children with unstable slips, female sex was a predisposing factor for developing AVN [10].

Concerning age distribution, the mean age of the patients at presentation was 12 years and this was in agreement with reports from other studies on the average age at presentation for female children [1,2]. This is the period of rapid growth spurt, thus predisposing high risk individuals to slips.

Concerning presenting complaints, all presented with hip pain as the typical nature of complaint except for one who presented with knee pain, comprising $14.3 \%$ of the study group. This is similar to the $15 \%$ reported by Matava et al. [11]. It was observed that there was a delay in diagnosing the patient who presented with knee pain. Such delays in similar circumstances have been reported in previous studies $[11,12]$ as such patients have pain referred from the hip to the knee. Thus, a high index of suspicion is required for adolescents presenting with knee pain, as such pain may be from the hip. 
The subcapital osteotomies have the greatest capacity to correct deformities resulting from physeal slip. This is because they are carried out at the Centre Of Rotation and Angulation (CORA). The Dunn and Fish osteotomies are the types of subcapital osteotomies employed in the period of review. Five hips had Dunn osteotomy while one had Fish osteotomy. They were all done via the anterior approach.

Our review had no patients with Chondrolysis or AVN as complications. This could be attributed to the open reduction and intraoperative imaging used during procedures. Early studies on subcapital osteotomy reported high rates of AVN (28.5\%) and chondrolysis (37.6\%) [13]. However, more recent studies on subcapital osteotomy done with surgical hip dislocation and intraoperative Imaging have shown low rates of AVN and chondrolysis. Ziebarth in his series of 40 patients had no AVN and no chondrolysis [14]. Alshryda et al. in their study comparing Fish osteotomy and PIS for severe SCFE had an AVN rate of $26.6 \%$ in the patients with unstable slips. They however explained that patients with unstable slips, who have been shown to have higher rates of $\mathrm{AVN}$, are more likely to have surgical reduction and osteotomy, indicating some degree of bias against the osteotomy group [15].

Subtrochanteric osteotomy makes use of a transverse osteotomy with a small wedge removed to achieve correction of residual deformity seen mostly in chronic SCFE. This correction aims to achieve valgus, internal rotation and flexion. It has been shown by Tjoumakaris et al to achieve good results when assessing outcomes such hip ROM, limp and radiographic results [16]. The failure to align ends of osteotomized bone, rather achieving a bayonet apposition had no influence on bone healing rates. Subtrochanteric osteotomy also has the advantage of being extracapsular, thereby eliminating the risk of osteonecrosis resulting for iatrogenic compromise of vascular supply to the femoral head. It is also particularly useful for correction of deformities in those who have had prior pinning.

\section{Conclusion}

The management of deformities arising from SCFE remain an area of controversy with regard to optimum treatment choice, and even when such a choice is obvious it's implementation is often technically challenging. Our experience as reflected in this study demonstrates that subcapital and subtrochanteric osteotomies appear to be excellent surgical options for the treatment of severe SCFE, with minimal risk of osteonecrosis and chondrolysis. Proper interventional studies with larger sample size would be required to address some of the questions raised herein.

\section{Limitations}

This is a retrospective review of a few patients with limited follow-up.

\section{Conflict of Interest}

The authors have no conflict of interest to declare and studies were done at no cost to the patients.

\section{References}

1. Novais EN, Millis MB (2012) Slipped capital femoral epiphysis: Prevalence, pathogenesis, and natural history. Clin Orthop Relat Res 470(12): 3432-3438.

2. Loder RT, Skopelja EN (2011) The Epidemiology and Demographics of Slipped Capital Femoral Epiphysis. ISRN Orthop 2011: 486512.

3. Benson EC, Miller M, Bosch P, Szalay EA (2008) A New Look at the Incidence of Slipped Capital Femoral Epiphysis in New Mexico. J Pediatr Orthop 28(5): 529-533.

4. Loder R, Richards S, Shapiro P, Reznick L, Aronson D (1993) Acute slipped capital femoral epiphysis: The importance of physeal stability. J Bone Joint Surg Am 75(8): 1134-1140.

5. Blom A, Warwick D, Whitehouse M (2018) Apley and Solomon's system of orthopaedics and fractures. 10th ed. CRC Pres Pp. 541-546.

6. Anderson LA, Gililland JM, Pelt CE, Peters CL (2013) Subcapital correction osteotomy for malunited slipped capital femoral epiphysis. J Pediatr Orthop 33(4): 345-352.

7. Yinusa W, Owoola A, Ahmed B (2010) In-situ pinning for Slipped Capital Femoral Epiphysis in blacks: experience in a regional orthopaedic centre. Niger Postgrad Med J 17(3): 190-193.

8. Katchy A (2017) Slipped Capital Femoral Epiphysis: A Review of 40 Consecutive Cases at The National Orthopaedic Hospital Enugu. Niger J Med 26(4): 334-337.

9. Shank CF, Thiel EJ, Klingele KE (2010) Valgus slipped capital femoral epiphysis: prevalence, presentation, and treatment options. J Pediatr Orthop 30(2): 140-146.

10. Palocaren T, Holmes L, Rogers K, Kumar SJ (2010) Outcome of in situ pinning in patients with unstable slipped capital femoral epiphysis: assessment of risk factors associated with avascular necrosis. J Pediatr Orthop 30(1): 31-36.

11. Matava M, Patton C, Luhmann S, Gordon J, Schoenecker P (1999) Knee pain as the initial symptom of slipped capital femoral epiphysis: an analysis of initial presentation and treatment. J Paediatr Orthop 19(4): 455-460.

12. Hosseinzadeh P, Iwinski HJ, Salava J, Oeffinger D (2017) Delay in the Diagnosis of Stable Slipped Capital Femoral Epiphysis. J Pediatr Orthop 37(1): e19-e22.

13. Gage James, Sundberg Bruce, Nolan Declan, Sletten RWR (1978) Complications after cuneiform osteotomy for moderately or severely slipped capital femoral epiphysis. J Bone Joint Surg Am 60(2): 157-165.

14. Ziebarth K, Zilkens C, Spencer S, Leunig M, Ganz R, et al. (2009) Capital realignment for moderate and severe SCFE using a modified dunn procedure. Clin Orthop Relat Res 467(3): 704-716.

15. Alshryda S, Tsnag K, Ahmed M, Adedapo A, Montgomery R (2014) Severe slipped upper femoral epiphysis; fish osteotomy versus pinning-in-situ: An eleven year perspective. Surgeon 12(5):244-248.

16. Tjoumakaris FP, Wallach DM, Davidson RS (2007) Subtrochanteric osteotomy effectively treats femoroacetabular impingement after slipped capital femoral epiphysis. Clin Orthop Relat Res 464: 230-237. 